Morphology and composition of oxidized InAs nanowires studied by combined Raman spectroscopy and transmission electron microscopy

This content has been downloaded from IOPscience. Please scroll down to see the full text. 2016 Nanotechnology 27305704

(http://iopscience.iop.org/0957-4484/27/30/305704)

View the table of contents for this issue, or go to the journal homepage for more

Download details:

IP Address: 128.178.120.129

This content was downloaded on 28/07/2016 at $11: 36$

Please note that terms and conditions apply. 


\title{
Morphology and composition of oxidized InAs nanowires studied by combined Raman spectroscopy and transmission electron microscopy
}

\author{
Rawa Tanta ${ }^{1}$, Thomas Kanne ${ }^{1}$, Francesca Amaduzzi ${ }^{2}$, Zhiyu Liao ${ }^{3}$, \\ Morten H Madsen ${ }^{4}$, Esther Alarcón-Lladó ${ }^{2}$, Peter Krogstrup ${ }^{1}$, \\ Erik Johnson ${ }^{1}$, Anna Fontcuberta i Morral ${ }^{2}$, Tom Vosch $^{3}$, \\ Jesper Nygård ${ }^{1}$ and Thomas S Jespersen ${ }^{1,5}$ \\ ${ }^{1}$ Center for Quantum Devices \& Nano Science Center, Niels Bohr Institute, University of Copenhagen, \\ Copenhagen, Denmark \\ ${ }^{2}$ Laboratory of Semiconductor Materials, Ecole Polytechnique Fédérale de Lausanne, 1015 Lausanne, \\ Switzerland \\ ${ }^{3}$ Nano-Science Center, Department of Chemistry, University of Copenhagen, Universitetsparken 5, DK- \\ 2100 Copenhagen, Denmark \\ ${ }^{4}$ Danish Fundamental Metrology A/S, Matematiktorvet 307, DK-2800 Kgs. Lyngby, Denmark \\ E-mail: tsand@fys.ku.dk
}

Received 25 January 2016, revised 27 April 2016

Accepted for publication 19 May 2016

Published 20 June 2016

\begin{abstract}
Any device exposed to ambient conditions will be prone to oxidation. This may be of particular importance for semiconductor nanowires because of the high surface-to-volume ratio and only little is known about the consequences of oxidation for these systems. Here, we study the properties of indium arsenide nanowires which were locally oxidized using a focused laser beam. Polarization dependent micro-Raman measurements confirmed the presence of crystalline arsenic, and transmission electron microscopy diffraction showed the presence of indium oxide. The surface dependence of the oxidation was investigated in branched nanowires grown along the [0001] and [01 10$]$ wurtzite crystal directions exhibiting different surface facets. The oxidation did not occur at the [0110] direction. The origin of this selectivity is discussed in terms transition state kinetics of the free surfaces of the different crystal families of the facets and numerical simulations of the laser induced heating.
\end{abstract}

Keywords: nanowires, oxidation, Raman, TEM

\section{Introduction}

Sparked by developments in crystal growth, semiconductor nanowires (NWs) have for the past decade been the focus of intense research in areas ranging from chemical and biological sensing [1], optical devices and solar cells [2-4], next generation electronics [5], and quantum devices [6, 7]. It is common for all applications of nanowires that devices will at some point be exposed to oxygen, and some degree of surface

\footnotetext{
5 Author to whom any correspondence should be addressed.
}

oxidation is inevitable. Because of the high surface-to-volume ratio of the nanowire geometry even small amounts of oxidation may have a large impact on the properties of the nanowires; effects that may be crucial for real application of nanowires where devices experience harsh environments and elevated temperatures. Investigating the fundamental oxidation process of the nanowires is therefore highly relevant. To this end, locally accelerating the reaction by focused laser irradiation has been demonstrated and can be combined with micro-Raman spectroscopy to provide information about the vibrational modes of individual semiconductor nanowires [8- 
11] and also conveying information about the elemental composition [12, 13], crystal properties [14], and defects [15] as well as free carrier densities and mobilities [16]. Also, at high intensities, focused laser irradiation has been shown to enable controlled local cutting or chemical modification of InAs and GaAs nanowires by local oxidation and formation of crystalline arsenic [17-21] and enables welding of metal nanowires [22]. This opens the possibility for using focused laser beams for simultaneous micro-Raman characterization and engineering new functionality into nanowire devices. So far, laser modified semiconductor nanowires have been characterized through their Raman spectrum and by atomic force microscopy, scanning electron microscopy and photoluminescence, however, the morphologies and crystal structures have not been studied. Here we show that $50 \mathrm{~nm}$ thick silicon nitride membranes can be used as an optimal substrate for correlating micro-Raman spectroscopy and transmission electron microscopy (TEM) imaging and we use this platform for analyzing the change in NW morphology and composition due to irradiation activated oxidation of InAs NWs. The results clearly show an amorphous NW surface coating and the presence of both crystalline arsenic and polycrystalline indium oxide thus providing support for previously proposed reaction schemes. The core of the NW remains intact as seen both from TEM and the Raman spectra. The polarization dependence of the Raman spectrum from the oxidized InAs NWs exhibits a strong enhancement of the Raman signal attributed to arsenic for parallel polarization in analogy to the antenna effect of pristine NWs [23]. Finally, using a unique branched NW structure we are able to compare directly two different surface facets. We show that the ability to locally induce modifications by laser irradiation depends strongly on the crystal orientation of the facets which can be controlled by changing the crystal direction of the NW growth.

\section{Methods}

The InAs NWs used in this work were grown by molecular beam epitaxy via the vapor-liquid-solid mechanism using gold particles randomly dispersed on planar substrates of (111)B InAs as growth catalysts. The NWs grew in the wurtzite crystal structure and two types of NWs have been investigated. The first type corresponds to standard InAs NWs with the [0001] crystal direction along the NW axis for details see [24]. These wires were grown using an As/In flux ratio of 20 and a corresponding planar growth rate of $0.7 \mu \mathrm{m} \mathrm{h}^{-1}$ with a substrate temperature of $T=435{ }^{\circ} \mathrm{C}$. The NWs are $\sim 7 \mu \mathrm{m}$ long, have hexagonal cross-sections and tapered morphologies with diameters ranging from $40 \mathrm{~nm}$ at the tip to $130 \mathrm{~nm}$ at the base. The NWs contain occasional random stacking-faults as seen in figure 1(b). To study the effect of crystal orientation and faceting, branched InAs NWs were grown with a V/III ratio of 40 and corresponding planar growth rate of $0.35 \mu \mathrm{m} \mathrm{h}^{-1}$ with a substrate temperature of $T=425^{\circ} \mathrm{C}$, in a two-step method: first, a conventional [0001] stem was grown and secondly, triggered by introducing a short Ga flux pulse, the catalyst gold droplet moved from the top to a side facet of
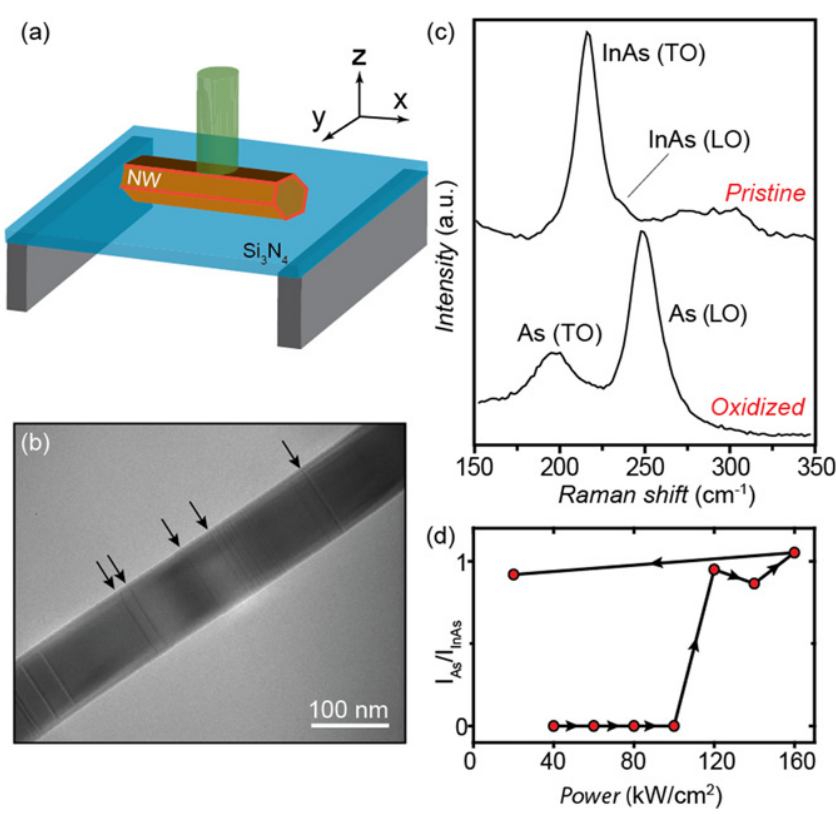

Figure 1. (a) Schematic illustration of the setup and the definition of the coordinate system. (b) TEM image of an InAs NW on a $50 \mathrm{~nm}$ thick $\mathrm{Si}_{3} \mathrm{~N}_{4}$ membrane. The NW is grown in the [0001] wurtzite crystal direction and arrows indicate occasional stacking faults. (c) Typical Raman spectra of pristine InAs NWs on Si substrate [21] (top curve), and oxidized InAs $\mathrm{NW}$ on a $\mathrm{Si}_{3} \mathrm{~N}_{4}$ membrane (bottom curve). Spectra are off-set for clarity. (d) Power dependence of the ratio of the arsenic LO and InAs TO Raman peaks for a NW suspended over a $1 \mu \mathrm{m}, 100 \mathrm{~nm}$ deep trench etched in silicon oxide. The arsenic peaks irreversibly appear at a threshold power of $120 \mathrm{~kW} \mathrm{~cm}^{-2}$ indicating the activation of the oxidation reaction.

the NW. A continuation of the growth resulted in axial growth of a branch along the [0110] crystal directions perpendicular to the [0001] see [25] for details. Typical growths have hexagonal [0001] stems of lengths $\sim 1 \mu \mathrm{m}$ and diameters of $120 \mathrm{~nm}$, and $\sim 2 \mu \mathrm{m}$ long [01 $\overline{1} 0]$ branches with diameters of $100 \mathrm{~nm}$ and trapezoidal cross-sections.

Samples were prepared for Raman and TEM measurements by ultrasonically suspending NWs from the growth substrate in isopropanol and depositing them either on substrates of $50 \mathrm{~nm}$ thick $\mathrm{Si}_{3} \mathrm{~N}_{4}$ membranes ${ }^{6}$ allowing a combination of TEM and Raman analysis, or on Si substrates with a $500 \mathrm{~nm}$ silicon oxide cap with or without chemically etched micro-trenches [21]. Figure 1(a) schematically shows the setup and introduces the coordinate system used in the following.

Raman spectra from individual InAs NWs were collected in a back-scattering geometry and two different home built setups were used. One used a $514.5 \mathrm{~nm}$ argon-ion laser (CVI Melles-Griot 35MAP431-200) and a power density of $450 \mathrm{~kW} \mathrm{~cm}^{-2}$, focused in an inverted confocal microscope by a $100 \times, 1.3$ NA oil immersion objective into a diffraction limited spot. A piezo-scanning stage (Physik Instrument P5173CL) allowed $x y$-positioning and scanning, and Raman spectra were collected using a Princeton Instruments SPEC-

6 PELCO $^{\circledR}, 21509-10$, silicon nitride support film, $50 \mathrm{~nm}$ with 9 each $0.1 \times 0.1 \mathrm{~mm}$ windows. 
10:100B/LN_eXcelon CCD detector and a SP 2356 spectrometer with $1200 \mathrm{~g} \mathrm{~mm}^{-1}$ grating blazed at $500 \mathrm{~nm}$. The polarization of the incident excitation was controlled by a motorized $\lambda / 2$ plate in the laser path before the microscope, and a linear polarizer in front of the spectrometer to select the polarization of the collected Raman scattered photons. The accumulation time of the spectra was $60 \mathrm{~s}$. In the second Raman setup, used for the measurements in figure 1(d), an $\mathrm{Ar}-\mathrm{Kr}^{+}$laser at $520.8 \mathrm{~nm}$ was focused on the NW by a microscope objective with numerical aperture NA $=0.75$. The scattered light was analyzed by a TriVista triple spectrometer and detected by a CCD detector. The oxidation effect was instantly induced by the Raman laser at power density $450 \mathrm{~kW} \mathrm{~cm}^{-2}$.

\section{Results and discussion}

Figure 1(b) shows an example of a TEM image of the NWs showing high crystal quality and occasional stacking faults, and figure 1(c) shows typical Raman spectra for the two cases of a pristine InAs NW on Si substrate [21] and an oxidized InAs NW on a TEM membrane. The spectra of the nonoxidized NW is identical to bulk InAs and contain one main peak at $\sim 216 \mathrm{~cm}^{-1}$ assigned to the InAs transverse optical (TO) phonon mode and a weak side-peak at $237 \mathrm{~cm}^{-1}$ assigned to the longitudinal optical (LO) mode. According to the Raman selection rules of the WZ crystal structure only the TO mode is allowed in this polarization configuration [26], however, the LO mode may appear due to crystal stacking faults in the NW [15]. As seen in the lowermost curve of figure $1(\mathrm{c})$ the oxidized NW shows a drastically different spectrum containing two high intensity peaks at 203 and $250 \mathrm{~cm}^{-1}$ which do not match any phonon energies of the InAs structure but rather the $E_{\mathrm{g}}(\mathrm{TO})$ mode at $198 \mathrm{~cm}^{-1}$ and $\mathrm{A}_{1 \mathrm{~g}}(\mathrm{LO})$ mode at $257 \mathrm{~cm}^{-1}$ of crystalline arsenic [27] which can form at the surfaces of InAs as a result of the reaction $\mathrm{As}_{2} \mathrm{O}_{3}+2 \mathrm{InAs} \rightarrow \mathrm{In}_{2} \mathrm{O}_{3}+4 \mathrm{As}$ as proposed in [28]. We note that the Raman peaks of indium oxide [29], at 231 and $304 \mathrm{~cm}^{-1}$, are absent in the spectrum consistent with previous investigations $[19,20,30]$. The oxidation reaction accelerates with increased temperature, and we attribute the appearance of the arsenic spectra as the result of laser induced heating [30, 31], possibly combined with photo-oxidation [28]. The activation of the oxidation reaction can be followed by the power dependence of the relative intensities of the fitted arsenic and InAs Raman peaks presented in figure 1(d) measured, using $520.8 \mathrm{~nm}$ laser excitation and $15 \mathrm{~min}$ integration time per spectrum, of a NW suspended over a $150 \mathrm{~nm}$ deep and $2 \mu \mathrm{m}$ wide trench [21]. Initially, at the lowest powers, the arsenic peaks are absent, but then irreversibly appear after applying illuminating powers above $120 \mathrm{~kW} \mathrm{~cm}^{-2}$.

The morphology and crystalline properties of the oxidized NWs can be assessed through TEM and polarized
Raman spectroscopy and may provide valuable information about the expected performance of NW devices having been exposed to severe treatment. Figures 2(b) and (c) show TEM images of a NW before and after irradiation. Initially, the NW has a uniform morphology but after the irradiation, the wire surface is covered by a low contrast coating and decorated by 5-10 nm grains of higher contrast. Figures 2(d) and (e) present a high-resolution TEM image and corresponding diffraction pattern for a different oxidized NW (see figure 3). The crystal planes of the NW are visible in the TEM image and the corresponding wurtzite InAs diffraction pattern in figure 2(e), confirms that a significant part of the crystalline wires remains intact. In addition, however, distinct ringshaped patterns appear in the diffraction pattern at positions consistent with the (222), (400) and (440) high intensity crystal planes of poly-crystalline $\mathrm{In}_{2} \mathrm{O}_{3}$. The presence of indium oxide has not previously been directly observed for irradiated NWs but is indeed the expected product of the reaction proposed in [28]. Interestingly, while the presence of crystalline arsenic is confirmed by the Raman spectra, the corresponding diffraction pattern is not observed in figure 2(e). This suggests that unlike the poly-crystalline $\mathrm{In}_{2} \mathrm{O}_{3}$, the arsenic appears as in [30] with a well-defined crystal orientation not allowing the diffraction in this particular orientation. The crystallinity of the arsenic may be further investigated through the polarization dependence of the Raman spectra as shown in figure 2(a). The spectrum with $z(x x) \bar{z}$ polarization configuration is identical to those in figure 1 and contains, in addition to the InAs $\mathrm{A}_{1}(\mathrm{TO})$ mode at $\sim 220 \mathrm{~cm}^{-1}$, the two arsenic peaks at $\sim 203$ and $\sim 255 \mathrm{~cm}^{-1}$, as expected from the wurtzite InAs and arsenic selection rules $[26,27]$. Interestingly, no trace of the arsenic modes appears for the $z(y y) \bar{z}$ and $z(y x) \bar{z}$ configurations, but appears again for the $z(x y) \bar{z}$ configuration. This is surprising, as the totally symmetric arsenic $\mathrm{A}_{1 \mathrm{~g}}(\mathrm{LO})$ mode should, according to the selection rules, appear for parallel polarization $(z(x x) \bar{z}$ and $z(y y) \bar{z})$ and not for crossed polarization $(z(x y) \bar{z}$ and $z(y x) \bar{z})$ and the arsenic TO mode should appear in all cases [27]. This expected polarization dependence was previously observed for oxidation induced on planar surfaces [30]. More detailed study is required in order to verify the origin of the observed effect. Due to the violation of the selection rules we could not conclude about the crystal orientation of the formed crystalline arsenic layer. These results, TEM and Raman, have been observed on all 10 measured NWs.

As confirmed by the morphology of the oxidized NWs in figure 2, the oxidation reaction occurs at the surface of the NW where oxygen is present. Thus, engineering and protecting the surface may be a way of controlling the reactions and it is therefore interesting to investigate the effect of oxidation on NWs with different surface facets. Figure 3 compares a NW grown in the conventional [0001] direction (having $\{01 \overline{1} 0\}$ surface facets) to a wire grown in the perpendicular [0110] direction (having $\{0001\} /\{11 \overline{2} 0\}$ surface facets). A schematic of the branched NW geometry is 
(a)

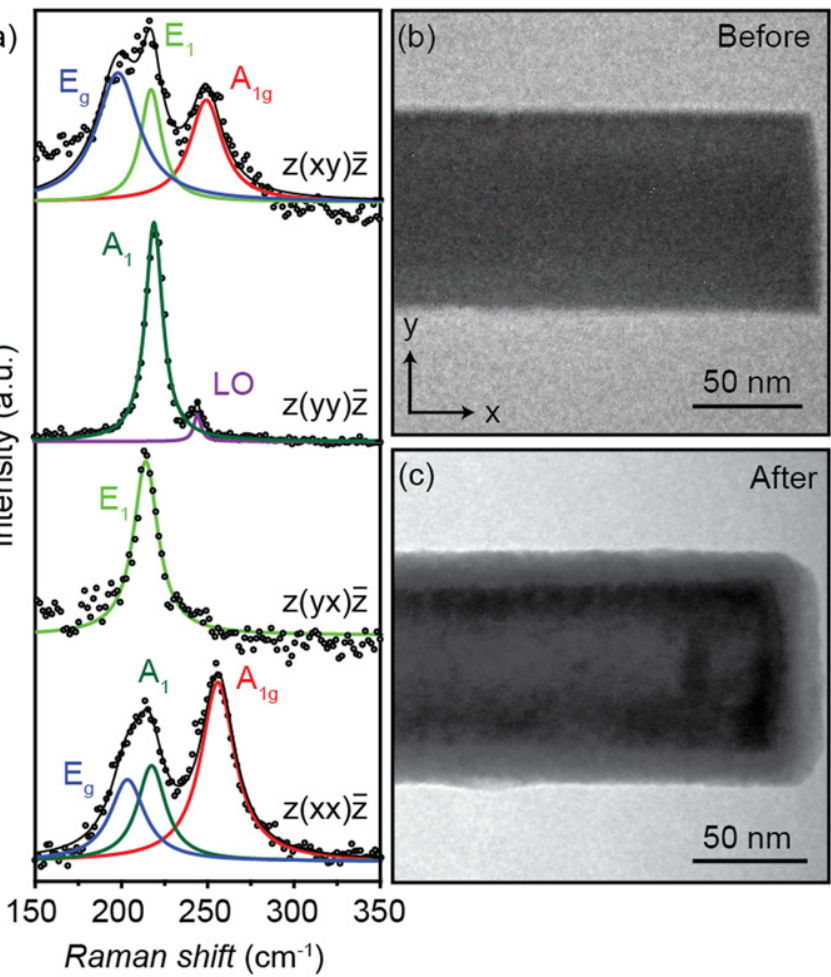

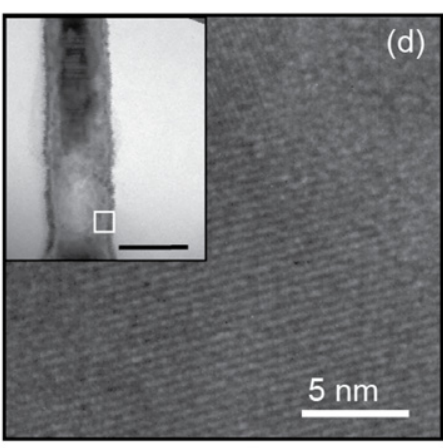

(d)

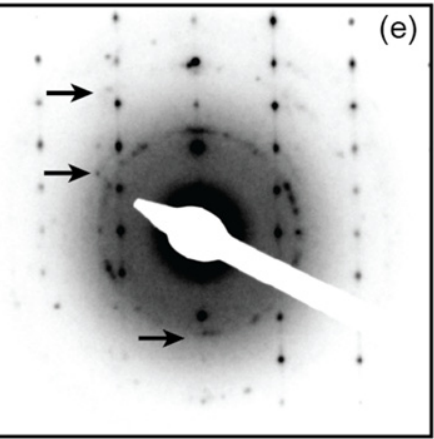

Figure 2. (a) Polarization dependent Raman spectra of InAs NW on $\mathrm{Si}_{3} \mathrm{~N}_{4}$ membrane. Spectra are fitted using a Lorentzian line shape and the InAs modes are observed at $220 \mathrm{~cm}^{-1}\left(\mathrm{~A}_{1}\right)$, at $216 \mathrm{~cm}^{-1}\left(\mathrm{E}_{1}\right)$, and $243 \mathrm{~cm}^{-1}(\mathrm{LO})$, whereas the arsenic modes are $\mathrm{E}_{\mathrm{g}}(\mathrm{TO})$ at $\sim 200 \mathrm{~cm}^{-1}$ and the $\mathrm{A}_{1 \mathrm{~g}}(\mathrm{LO})$ at $\sim 253 \mathrm{~cm}^{-1}$. (b), (c) TEM images of the measured NW bottom end, recorded before and after Raman measurements, respectively. (d) and (e) are TEM image and selected area diffraction of a different NW. The arrows in (e) indicate diffraction rings corresponding to poly-crystalline $\mathrm{In}_{2} \mathrm{O}_{3}$.

(a)

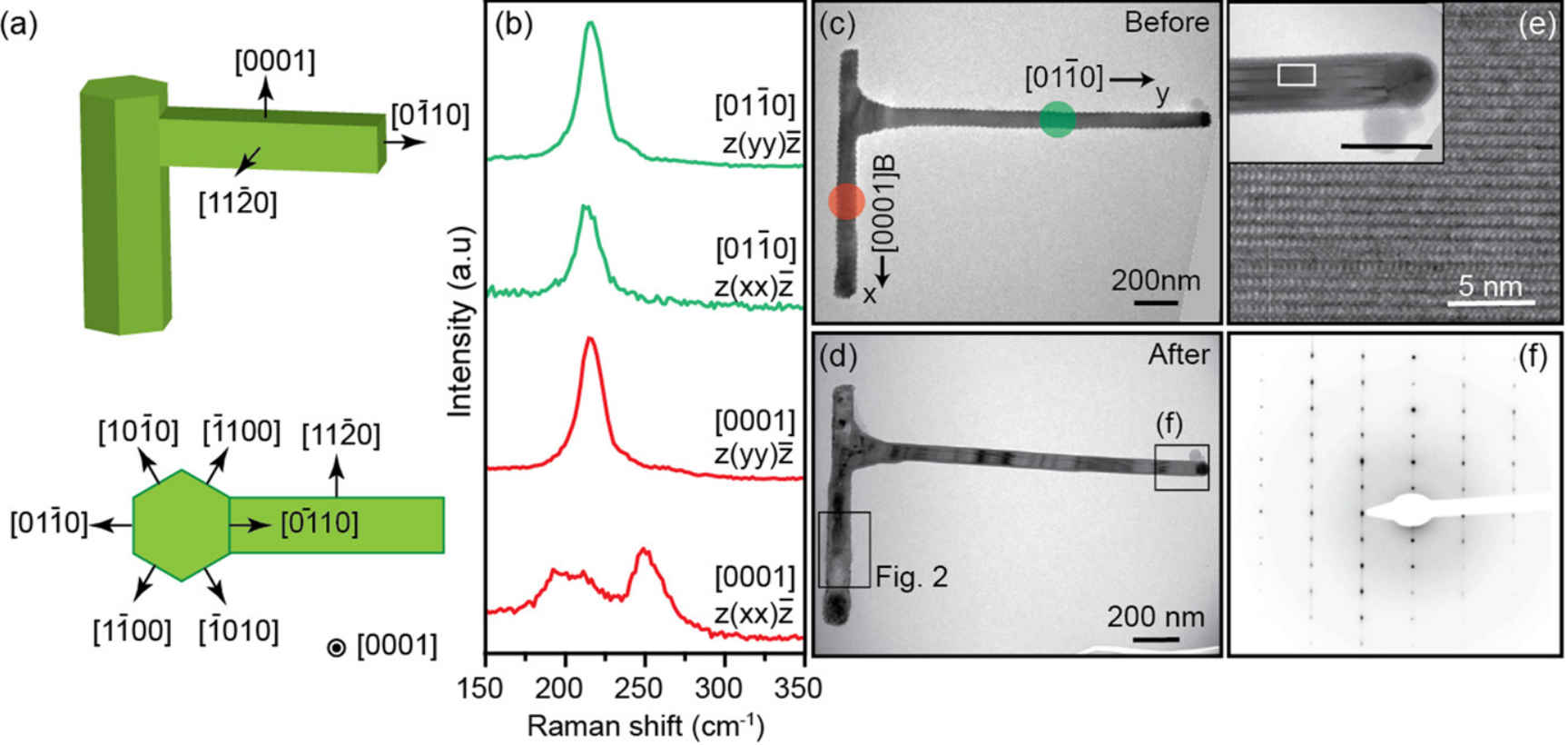

Figure 3. (a) A schematic illustrating the geometry of the branched NW structure. (b) Raman spectra at the [0110] and [0001] segments with the indicated polarization configurations. (c) and (d) TEM images of the NW measured in (b) before and after irradiation, respectively. Green and red circles indicate position of the spectra in (b). (e), (f) High resolution TEM images and selected area diffraction of the regions indicated in (d). The scale bar in the inserts is $100 \mathrm{~nm}$. 
presented in figure 3(a) and a typical structure is shown in the TEM image in figure 3(c) prior to the laser exposure. The [0001] stem and the [0110] branch are identified by the morphology of the structure and the position of the gold catalyst particle at the end of the branch.

Figure 3(b) shows Raman spectra measured at the stem and branch for both parallel and perpendicular polarizations as indicated. As in figure 2, the arsenic peaks are observed for the [0001] stem and only for parallel polarization, however, interestingly these peaks are entirely absent in the [0110] branch. The TEM and diffraction pattern of the [0001] stem are presented in figures $2(\mathrm{~d})$ and (e) clearly showing oxidation, while the corresponding measurements for the branch in figures 3(e) and (f) show no change of the NW morphology and contain no $\operatorname{In}_{2} \mathrm{O}_{3}$ signal in the diffraction pattern and thus confirm that the $[01 \overline{1} 0] \mathrm{NW}$ remained unaffected by the irradiation. This striking dependence on growth direction, which was observed in all five measured NWs, may be related to the dependence of the stabilities of the surface facets, or may be an effect of the different geometric cross sections of the structure possibly modifying the light absorption and heat dissipation. In the following we address these possibilities individually ${ }^{7}$.

The oxidation process consists of several parallel reaction schemes, where each scheme typically includes two sequential reactions [19, 28]. Let us first consider the oxidation reaction kinetics of InAs surfaces in general terms, valid for all possible reactions including InAs. Instead of identifying a specific rate limiting reaction, we thus consider the irreversible transition rates $\Gamma^{\mathrm{ox}}$ of a general oxidation reaction of InAs, which can be written as [32]:

$$
\Gamma^{\mathrm{ox}} \propto c_{\text {oxide }} c_{\text {InAs }} \exp \left[-\frac{\sum_{A} \delta h^{A}-\sum_{A, B} \delta \mu_{B}^{A}}{k_{B} T}\right] .
$$

Here $c_{\text {InAs }}$ and $c_{\text {oxide }}$ are the interfacial concentrations, and $\delta h^{A} \approx U^{A}-\gamma_{j}^{A}$ is the activation energy which is the energy required to dissociate an atom at the interface $j$ of phase $A$ with respect to a reference state. $U^{A}$ is the bulk cohesive energy, $\gamma_{j}^{A}$ is the interface energy excess per surface atom contributing with dangling bonds and represents a relative measure of the surface stability. $\delta \mu_{B}^{A}$ is the relative chemical potential (i.e. the thermodynamic driving force for the reaction) of element $B$ (As, $\mathrm{O}$ or $\mathrm{In}$ ) in phase $A$ (such as: $\mathrm{As}_{2} \mathrm{O}_{3}$, InAs, $\mathrm{In}_{2} \mathrm{O}_{3}, \mathrm{InAsO}_{4}$ ) relative to the reference state and is defined as:

$$
\delta \mu_{B}^{A}=\delta \mu_{B}^{A, \text { bulk }}+\gamma_{j} \frac{\partial A_{j}}{\partial n_{B}}+A_{j} \frac{\partial \gamma_{j}}{\partial n_{B}},
$$

where the first term relates to the bulk free energy (which we are not interested in here because it is the same for both types of wires) and the other two terms relate to the excess free energy associated with the interfaces. $A_{j}$ is the area of the interface $j$, and $n_{B}$ is the number of atoms $B$ in the phase $A$.

\footnotetext{
7 Elementary Ga is present on the stem due to the initiation of the kink during growth. This could have a catalyzing effect on the oxidation process, but was neglected since the same effect was observed on pure [0001] InAs nanowires.
}

Because the oxides are poly-crystalline with a dominant crystal direction, and because the reaction schemes are the same on the kink and on the stem, the only difference in the exponential of equation (1) is either $\gamma_{j}^{\text {InAs }}$ (in the activation energy $\delta h^{A}$ ) or the size dependent part of the chemical potentials $\gamma_{j}^{\text {InAs }} \frac{\partial A_{j}}{\partial n_{B}}$ which is negligible due to the scale of the NWs. The third term in equation (2) is also negligible assuming the same response to change in surface energy at the two directions. This means that the difference seen in the oxidation rates is solely a kinetic effect $\left(\delta h^{A}\right)$ rather than driven by thermodynamic driving forces $\left(\delta \mu_{B}^{A}\right)$. At thermal equilibrium, the differences in transition rates of the two types of facets are caused by differences in surface stabilities, with a reaction rate ratio given by $\Gamma_{01 \overline{1} 0}^{\mathrm{ox}} / \Gamma_{11 \overline{2} 0}^{\mathrm{ox}} \propto \exp \left[\frac{\gamma_{0110}^{\mathrm{InAs}}-\gamma_{1120}^{\mathrm{InAs}}}{K_{B} T}\right]$ using equation (1). However, implementing reported values for surface energies in the literature [33], which is a measure of $\gamma_{j}^{A}$, do not show any significant differences between the stability of $\{01 \overline{1} 0\}$ facets of the stem and the $\{11 \overline{2} 0\}$ facets of the kink.

Alternatively, differences in absorption efficiency or in thermal dissipation could be a consequence of the differences in geometry of the two types of NWs and thereby explain the observed selectivity. To investigate the possible differences in temperature, we simulated light absorption for the two geometries using the finite-difference time-domain package MEEP [34] treating the incident light as a plane wave polarized along the NW axis. For the absorption efficiency, we calculated the flux inside a box around the NW given by a top plane wave incoming onto it [35] with reference to a simulation performed without the NW. The flux through the projected area of the NW on the bottom face of the box was calculated. The simulated internal field distributions are shown in figures 4(a) and (b) and the resulting values of the absorption efficiency were $165 \%$ and $157 \%$ for the [01 10 ] and [0001] NWs, respectively, which does not explain the observed differences in oxidation.

While the main source of heat dissipation from nanostructures at these temperatures are through radiation, temperature differences of the two types of NWs may also be related to different areas for dissipating heat through the substrate. To investigate the magnitude of this effect, the temperature profiles of the irradiated NWs on a $50 \mathrm{~nm}$ thick $\mathrm{Si}_{3} \mathrm{~N}_{5}$ substrate were simulated using COMSOL Multiphysics as shown in figures 4(c)-(f) for various position of the effective heat source and areas of nanowire-substrate interface $^{8}$. Because the $[01 \overline{1} 0] \mathrm{NW}$ always originates from a facet of the [0001] NW in the branched structure, the orientation of the [0001] segment on the substrate is most likely as in figure 4(c) while it will be as figure 4(f) for non-branched NW. In both cases we observe oxidation of the [0001] NW. The conclusion from the simulations is that for the same position of the power source, as in figures 4(d)-(f), the temperature of the NW surface decreases when increasing the

8 The relatively low temperature scale ( $<350 \mathrm{C}$ oxidation threshold) which is a consequence of the substrtae effect on the simulations and since it is used for comparison can still be considered. 

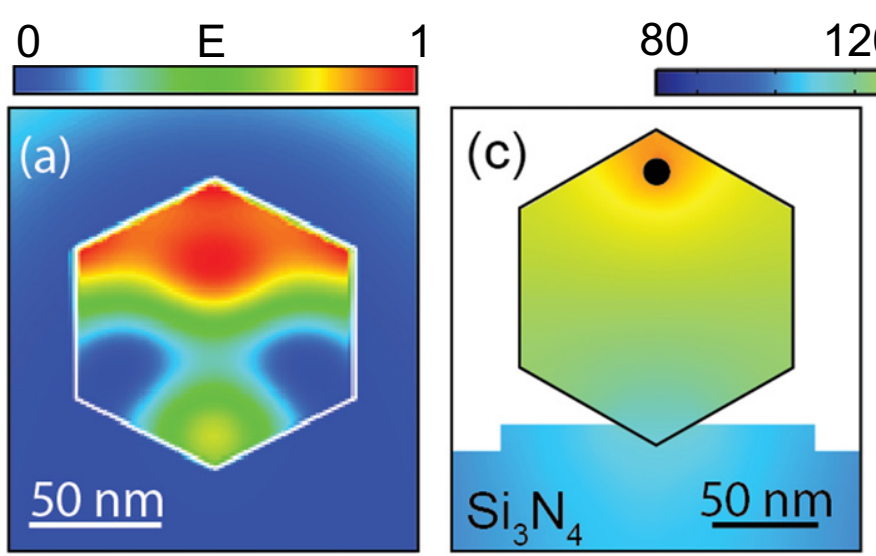

$20 \quad 160$
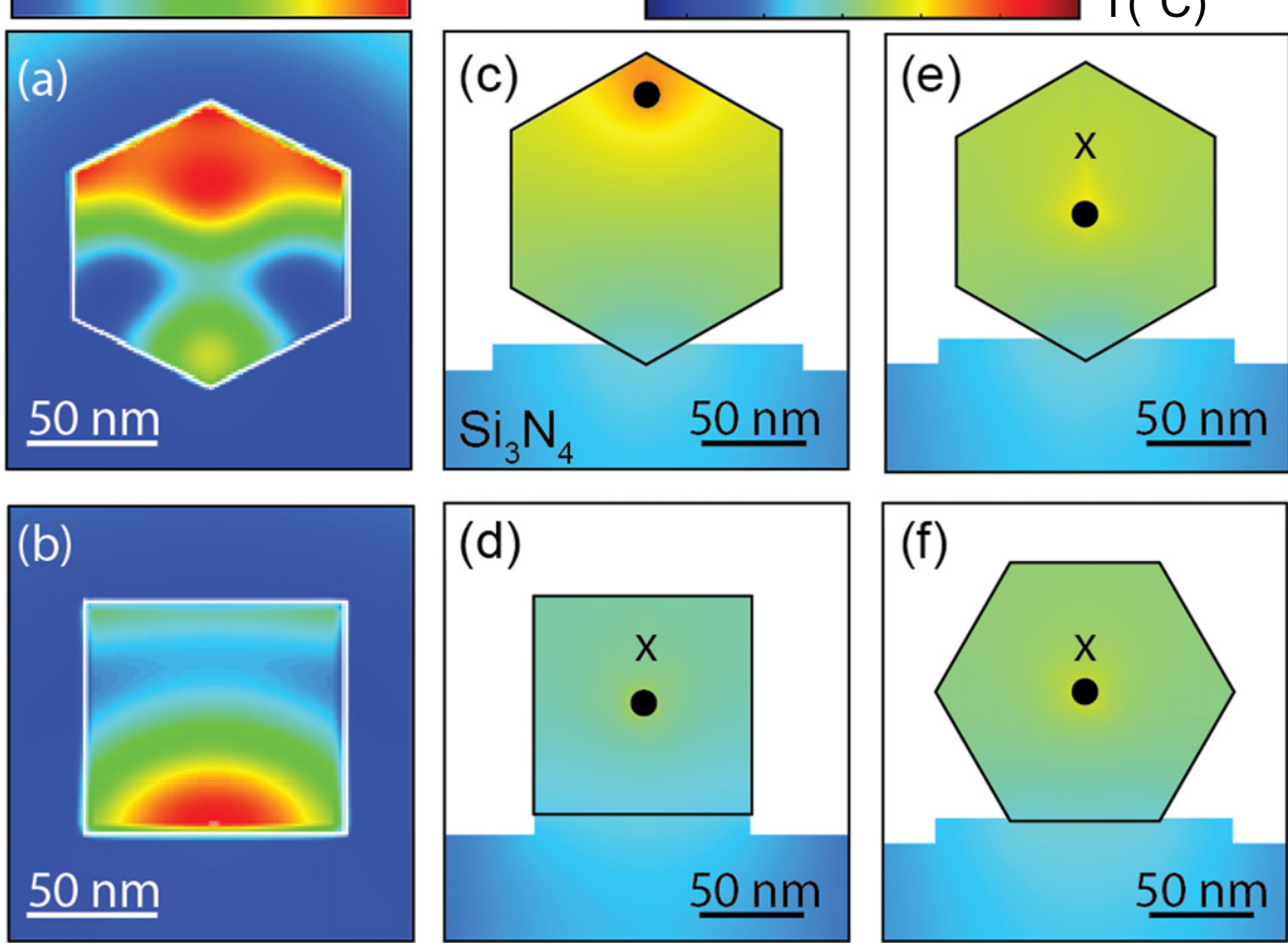

Figure 4. (a) and (b) Cross-sectional maps of the normalized field energy distribution for the [0001] stem and the [01 10$]$ kink, respectively. (c)-(f) Temperature profiles for different position of the heat source (black circle) and NW orientations on the substrate. The temperature at the positions marked by crosses in panels (d)-(f) are $115^{\circ} \mathrm{C}, 120^{\circ} \mathrm{C}$, and $130{ }^{\circ} \mathrm{C}$, respectively.

contact area, however, the $15^{\circ} \mathrm{C}$ lower temperature at the [0110] NW compared to the stem is insufficient to explain the selectivity in oxidation. In the simulations, the thermal conductivities were assumed identical in the two directions in order to show the effects of the different NW geometries. For ZB NW, however, numerical studies by Zhou et al [36] show that the thermal conductivity along [110] direction is $\sim 3$ times larger than along the [111] or [100] direction. Similar calculation for the WZ phase does not exist, however, experimental studies show that for the $[111] /[0001]$ directions, the WZ and ZB crystal phases have similar thermal conductivities [37]. Thus a possible explanation of the observed selectivity is an enhanced thermal conductivity along the branch directions leading to a lower temperature.

\section{Conclusions}

In conclusion, we have studied the surface oxidation process of InAs NWs by combining polarized micro-Raman spectroscopy and TEM on individual InAs NWs, having been irradiated by a high power laser to promote oxidation. By TEM diffraction we show directly for the first time the presence of indium oxide, and the Raman spectra confirm the presence of crystalline arsenic on the wire-oxide interface. The TEM analysis confirmed that the oxidation process occurs at the NW surface and that the InAs crystal remained in the NW core. Thus the oxide may provide a protecting self- passivation for NW devices functioning at highly oxidizing environments, and conversely, irradiation by a focused laser beam may provide a tool for locally effective thinning of NWs thereby inducing local regions with modified properties such as electrical barriers in electrical devices or increased sensitivity to the immediate chemical environment. Finally, we studied the dependence of the laser induced modification on the crystal direction of the InAs NW finding that NWs grown along unconventional directions $01 \overline{1} 0$ are more robust towards high power laser irradiation. The results show that the oxidation efficiency is strongly dependent on the NW crystal structure and morphology. This observation was discussed in terms of transition state kinetics of NW facets and simulation of the role of NW geometry for light absorption efficiency and heat dissipation. The simulations were not conclusive regarding the origin of the observed oxidation selectivity which we finally attributed to the anisotropy of the thermal conductivity along the NW. However the results suggest that engineering the substrate-nanowire interactions may provide a route for locally controlling the oxidation reactions on the surface of the NWs.

\section{Acknowledgments}

We thank the Danish Agency for Science Technology and Innovation (The Danish Council for Strategic Research -ANaCell project), 'Center for Synthetic Biology' at 
Copenhagen University funded by the UNIK research initiative of the Danish Ministry of Science, Technology and Innovation (Grant 09-065274), bioSYNergy, University of Copenhagen's Excellence Programme for Interdisciplinary Research, the Lundbeck Foundation, the Carlsberg Foundation, the ERC Stg 'UpCon' and SNF through project $\mathrm{nr}$ 156081. The Center for Quantum Devices is supported by the Danish National Research Foundation.

\section{References}

[1] Duan X and Lieber C M 2015 Nanoscience and the nanobioelectronics frontier Nano Res. 8 1-22

[2] Duan X, Huang Y, Cui Y, Wang J and Lieber C M 2001 Indium phosphide nanowires as building blocks for nanoscale electronic and optoelectronic devices Nature 409 66-9

[3] Li Y, Qian F, Xiang J and Lieber C M 2006 Nanowire electronic and optoelectronic devices Mater. Today 9 18-27

[4] Krogstrup P, Jørgensen H I, Heiss M, Demichel O, Holm J V, Aagesen M, Nygard J and Fontcuberta i Morral A 2013 Single-nanowire solar cells beyond the Shockley-Queisser limit Nat. Photon. 7 306-10

[5] Thelander C et al 2006 Nanowire-based one-dimensional electronics Mater. Today 9 28-35

[6] Hofstetter L, Csonka S, Nygård J and Schönenberger C 2009 Cooper pair splitter realized in a two-quantum-dot Y-junction Nature 461 960-3

[7] Mourik V, Zuo K, Frolov S M, Plissard S R, Bakkers E P A M and Kouwenhoven L P 2012 Signatures of Majorana fermions in hybrid superconductor-semiconductor nanowire devices Science 336 1003-7

[8] Cantoro M, Klekachev a V, Nourbakhsh A, Sore'e B, Heyns M M and De Gendt S 2011 Long-wavelength, confined optical phonons in InAs nanowires probed by Raman spectroscopy Eur. Phys. J. B 79 423-8

[9] Zardo I, Yazji S, Hörmann N, Hertenberger S, Funk S, Mangialardo S, Morkötter S, Koblmüller G, Postorino P and Abstreiter G 2013 E1(A) electronic band gap in wurtzite InAs nanowires studied by resonant Raman scattering Nano Lett. 13 3011-6

[10] Begum N, Piccin M, Jabeen F, Bais G, Rubini S, Martelli F and Bhatti A S 2008 Structural characterization of GaAs and InAs nanowires by means of Raman spectroscopy J. Appl. Phys. 104104311

[11] Kumar Panda J, Roy A, Singha A, Gemmi M, Ercolani D, Pellegrini V and Sorba L 2012 Raman sensitivity to crystal structure in InAs nanowires Appl. Phys. Lett. 100143101

[12] Stefaniak E A, Buczynska A, Novakovic V, Kuduk R and Grieken R V 2009 Determination of chemical composition of individual airborne particles by SEM/EDX and microRaman spectrometry: a review J. Phys.: Conf. Ser. 162 012019

[13] Majzner K, Kochan K, Kachamakova-Trojanowska N, Maslak E, Chlopicki S and Baranska M 2014 Raman imaging providing insights into chemical composition of lipid droplets of different size and origin: in hepatocytes and endothelium Anal. Chem. 86 6666-74

[14] Millot M, Gonzalez J, Molina I, Salas B, Golacki Z, Broto J M, Rakoto H and Goiran M 2006 Raman spectroscopy and magnetic properties of bulk $\mathrm{ZnO}$ :Co single crystal J. Alloys Compd. 423 224-7

[15] Zardo I, Conesa-Boj S, Peiro F, Morante J R, Arbiol J, Uccelli E, Abstreiter G and Fontcuberta i Morral A 2009
Raman spectroscopy of wurtzite and zinc-blende GaAs nanowires: polarization dependence, selection rules, and strain effects Phys. Rev. B 80245324

[16] Ketterer B, Uccelli E and Fontcuberta i Morral A 2012 Mobility and carrier density in p-type GaAs nanowires measured by transmission Raman spectroscopy Nanoscale 4 1789-93

[17] He J, Chen P, Lu W, Dai N and Zhu D-M 2012 Laser induced modification and ablation of InAs nanowires J. Appl. Phys. 111094316

[18] Lu J, Liu H, Zheng M, Zhang H, Lim S X, Tok E S and Sow C H 2014 Laser modified ZnO/CdSSe core-shell nanowire arrays for micro-steganography and improved photoconduction Sci. Rep. 46350

[19] Pal S, Aggarwal R, Kumari Gupta V and Ingale A 2014 Time evolution studies of laser induced chemical changes in InAs nanowire using Raman spectroscopy Appl. Phys. Lett. 105 012110

[20] Yazji S, Zardo I, Soini M, Postorino P, Fontcuberta i Morral A and Abstreiter G 2011 Local modification of GaAs nanowires induced by laser heating Nanotechnology 22325701

[21] Tanta R, Madsen M H, Liao Z, Krogstrup P, Vosch T, Nygård J and Jespersen T S 2015 Raman spectroscopy and electrical properties of InAs nanowires with local oxidation enabled by substrate micro-trenches and laser irradiation Appl. Phys. Lett. 107243101

[22] Garnett E C, Cai W, Cha J J, Mahmood F, Connor S T, Greyson Christoforo M, Cui Y, McGehee M D and Brongersma M L 2012 Self-limited plasmonic welding of silver nanowire junctions Nat. Mater. 11 241-9

[23] Xiong Q, Chen G, Gutierrez H and Eklund P 2006 Raman scattering studies of individual polar semiconducting nanowires: phonon splitting and antenna effects Appl. Phys. A 85 299-305

[24] Madsen M H, Krogstrup P, Johnson E, Venkatesan S, Mühlbauer E, Scheu C, Sørensen C B and Nygård J 2013 Experimental determination of adatom diffusion lengths for growth of InAs nanowires J. Cryst. Growth 364 16-22

[25] Krogstrup P, Ziino N L B, Chang W, Albrecht S M, Madsen M H, Johnson E, Nygård J, Marcus C M and Jespersen T S 2015 Epitaxy of semiconductorsuperconductor nanowires Nat. Mater. 14 400-6

[26] Möller M, De Lima M M, Cantarero a, Dacal L C O, Madureira J R, Iikawa F, Chiaramonte T and Cotta M a 2011 Polarized and resonant Raman spectroscopy on single InAs nanowires Phys. Rev. B 84085318

[27] Renucci J B, Richter W, Cardona M and Schostherr E 1973 Resonance raman scattering in group Vb semimetals: As, Sb, and Bi Phys. Status Solidi 60 299-308

[28] Hollinger G, Skheyta-Kabbani R and Gendry M 1994 Oxides on GaAs and InAs surfaces: an x-ray-photoelectronspectroscopy study of reference compounds and thin oxide layers Phys. Rev. B 49 11159-67

[29] Wang J X et al 2005 Synthesis and characterization of In2O3/ $\mathrm{SnO} 2$ hetero-junction beaded nanowires J. Cryst. Growth 284 73-9

[30] Farrow R L, Chang R K, Mroczkowski S and Pollak F H 1977 Detection of excess crystalline As and Sb in III-V oxide interfaces by Raman scattering Appl. Phys. Lett. 31768

[31] Piscanec S, Cantoro M, Ferrari A, Zapien J, Lifshitz Y, Lee S, Hofmann S and Robertson J 2003 Raman spectroscopy of silicon nanowires Phys. Rev. B 68241312

[32] Krogstrup P, Jørgensen H I, Johnson E, Madsen M H, Sørensen C B, Morral A F i, Aagesen M, Nygård J and Glas F 2013 Advances in the theory of III-V nanowire growth dynamics J. Phys. D: Appl. Phys. 46313001 
[33] Sibirev N V, Timofeeva M A, Bol'shakov A D,

Nazarenko M V and Dubrovskiĭ V G 2010 Surface energy and crystal structure of nanowhiskers of III-V

semiconductor compounds Phys. Solid State 52 1531-8

[34] Oskooi A F, Roundy D, Ibanescu M, Bermel P,

Joannopoulos J D and Johnson S G 2010 Meep: a flexible free-software package for electromagnetic simulations by the FDTD method Comput. Phys. Commun. 181 $687-702$
[35] Heiss M and Fontcuberta i Morral A 2011 Fundamental limits in the external quantum efficiency of single nanowire solar cells Appl. Phys. Lett. 99263102

[36] Zhou W-X, Chen K-Q, Tang L-M and Yao L-J 2013 Phonon thermal transport in InAs nanowires with different size and growth directions Phys. Lett. A 377 3144-7

[37] Zhou F et al 2011 Thermal conductivity of indium arsenide nanowires with wurtzite and zinc blende phases Phys. Rev. B 83205416 